\title{
SANCDB: an update on South African natural compounds and their readily available analogs
}

\author{
Bakary N'tji Diallo', Michael Glenister ${ }^{1}$, Thommas M. Musyoka ${ }^{1}$, Kevin Lobb ${ }^{1,2}$ and Özlem Tastan Bishop ${ }^{1 *}$ (I)
}

\begin{abstract}
Background: South African Natural Compounds Database (SANCDB; https://sancdb.rubi.ru.ac.za/) is the sole and a fully referenced database of natural chemical compounds of South African biodiversity. It is freely available, and since its inception in 2015, the database has become an important resource to several studies. Its content has been: used as training data for machine learning models; incorporated to larger databases; and utilized in drug discovery studies for hit identifications.

Description: Here, we report the updated version of SANCDB. The new version includes 412 additional compounds that have been reported since 2015, giving a total of 1012 compounds in the database. Further, although natural products (NPs) are an important source of unique scaffolds, they have a major drawback due to their complex structure resulting in low synthetic feasibility in the laboratory. With this in mind, SANCDB is, now, updated to provide direct links to commercially available analogs from two major chemical databases namely Mcule and MolPort. To our knowledge, this feature is not available in other NP databases. Additionally, for easier access to information by users, the database and website interface were updated. The compounds are now downloadable in many different chemical formats.

Conclusions: The drug discovery process relies heavily on NPs due to their unique chemical organization. This has inspired the establishment of numerous NP chemical databases. With the emergence of newer chemoinformatic technologies, existing chemical databases require constant updates to facilitate information accessibility and integration by users. Besides increasing the NPs compound content, the updated SANCDB allows users to access the individual compounds (if available) or their analogs from commercial databases seamlessly.
\end{abstract}

Keywords: SANCDB, Natural products, Commercial analogs, Drug discovery

\section{Introduction}

Throughout history, natural products (NPs) have benefited mankind in food, pesticides, cosmetic products and as drugs [1]. NP research has, especially, been a growing field in modern drug discovery as they offer unique chemical scaffolds [2], hence a greater structural diversity than synthetic ones, and they cover a large area of

\footnotetext{
*Correspondence: O.tastanbishop@ru.ac.za

${ }^{1}$ Research Unit in Bioinformatics (RUBi), Department of Biochemistry and Microbiology, Rhodes University, Makhanda/Grahamstown 6140, South Africa

Full list of author information is available at the end of the article
}

chemical space [3]. Between 2010 and 2019, NPs contributed to $25-33 \%$ of approved small molecules [4], and it is estimated that they represent $35 \%$ of medicines [5]. Some of the notable approved drugs, either from pure or derived NPs, include lefamulin, the aminoglycoside antibiotic plazomicin; tafenoquine succinate, an antimalarial agent; and aplidine, an anticancer agent [4]. Given the high interest in NP research, over 120 NP databases and collections have been developed, and are continuously being updated $[1,3]$ with new information and functionalities.

The South African Natural Compounds Database (SANCDB; https://sancdb.rubi.ru.ac.za/) is a fully

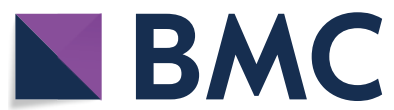

(c) The Author(s) 2021. This article is licensed under a Creative Commons Attribution 4.0 International License, which permits use, sharing, adaptation, distribution and reproduction in any medium or format, as long as you give appropriate credit to the original author(s) and the source, provide a link to the Creative Commons licence, and indicate if changes were made. The images or other third party material in this article are included in the article's Creative Commons licence, unless indicated otherwise in a credit line to the material. If material is not included in the article's Creative Commons licence and your intended use is not permitted by statutory regulation or exceeds the permitted use, you will need to obtain permission directly from the copyright holder. To view a copy of this licence, visit http://creativeco mmons.org/licenses/by/4.0/. The Creative Commons Public Domain Dedication waiver (http://creativecommons.org/publicdomain/ zero/1.0/) applies to the data made available in this article, unless otherwise stated in a credit line to the data. 
referenced database of NPs derived from sources within South Africa [6]. The database and website were established by the Research Unit in Bioinformatics (RUBi) in 2015. The main content of the database is a set of NP chemical structures in different chemical formats linked to their primary literature reference. Since its creation, the database has attracted significant interest in diverse domains including NP research, drug discovery, cheminformatics and machine learning with up to 52 citations. Besides its use in hit identification in drug discovery studies, SANCDB content has also served as training data for machine learning models [7, 8]; training data for a NPs likeness scorer (NaPLeS) [7]; and for NP-Scout, a machine learning approach for the identification of NPs [9]. Similarly, the database compounds have served to train STarFish, a target fishing model for NPs [8]. SANCDB was also utilized as an intermediate node for data integration into larger or more specialized information systems. An example is Natural Product Activity and Species Source (NPASS), which is a NP activity and species source database built on some NPs data resources, including SANCDB [10]. Similarly, SANCDB data has also been used in the COCONUT (COlleCtion of Open Natural ProdUcTs) database [11].

The usage of the South African natural compounds as drugs is yet to be reported. However, the search for potential hits with activity against infectious agents and cancer is on the rise, and may lead to the identification of drugs in the near future [12]. In terms of drug discovery studies, the database has been used for identification of hit compounds against the active (orthosteric) site of various biological drug targets in diseases including malaria, trypanosomiasis and severe acute respiratory syndrome Corona Virus 2 (SARS-COV-2) [13-18]. Additionally, potential allosteric modulators such as 20(29)-lupene$3 \beta$-isoferulate (SANC00518) for human Hsp90 $\alpha$ [14]; discorhabdin N (SANC00132), for human Hsp72 and Hsc70 [15]; gordonoside A (SANC00456) for Plasmodium falciparum Prolyl tRNA synthetase [19] have also been identified from SANCDB.

Here, we present an updated version of SANCDB with a number of new features. Firstly, over the last five years, since the inception of SANCDB, considerable NP research has been performed in the country. For instance, in 2019, Fantoukh et al. isolated 11 compounds from Aspalathus linearis [20], and Awolola et al. reported four compounds from the genus Ficus [21]. A variety of flavonoids, proanthocyanidins, ellagitannins, oligosaccharides and quinic acid derivatives were isolated from Myrothamnus flabellifolia Welw in 2016 [22]. Thus, the updated SANCDB has curated such continuously growing information. Secondly, in the updated version of the database, we provide links to commercially available analogs for each compound to increase the search space and the availability of physical compounds. We hope that this unique feature of SANCDB will later be implemented by other NPs databases. Most of the time NPs' physical availability is either limited [1] or very expensive due to required isolation methods [5,23]; and it has been well demonstrated that they can serve as good start points for the development of synthesizable analogs which may lead to effective drugs [24]. Finally, we include a scaffold analysis for all compound entries; this was undertaken to determine the database chemical diversity, which is an important aspect in the exploration of potential hit compounds [25]. Compound classification in SANCDB is also revisited.

\section{Methods}

\section{Update of the database}

NPs isolated from South African sources were searched in the literature, and uploaded through the earlier pipeline described in the preceding publication [6]. Elsevier's abstract and citation database (Scopus) Application Programming Interface (API) was used to identify more references. This allowed access to the scholarly databases indexed by Scopus [26] for collection, parsing and extraction of organized literature references. From the current set of references in SANCDB, we retrieved the list of all authors using the reference Digital Object Identifier (DOI). Using the Scopus API [26], a list of all publications associated with each author was retrieved. Both redundant publications and those in which none of the authors had a South African affiliation were removed as a pre-filtering step. From the remaining list of publications, the abstracts, and if available, the full text of the articles, were retrieved. Keywords "South Africa", "compound" and "isolate" were then searched in the abstracts and full texts, removing documents not presenting any of these keywords. The resulting list of publications was then searched in SciFinder [27] to find each compound's Chemical Abstracts Service (CAS) [27] number. References were then checked to confirm that sources were indeed from South Africa. Using an updated pipeline described in the original publication [6], new compounds were uploaded into the database. Compound sources were mapped to their genera, families and kingdoms using pygbif [28] a Python client for the Global Biodiversity Information Facility (GBIF) [29] API. PubChemPy [30] was used to retrieve additional information on chemical compounds utilizing their CAS number as query. Compound IDs for different databases (ChEMBL [31], DrugBank [32], ZINC [33], PubChem [34]) were automatically retrieved from PubChem [34]. Besides the previously assigned compound classification generated manually, additional automated classification based on 
ClassyFire [35] was also included. To allow diverse usage of the compounds in docking studies, structures were prepared in different ready-to-dock formats, viz AutoDock pdbqt and Schrödinger Maestro format [36]. All aromatic compound structure depictions were standardized to the Kekulé form.

\section{Analogs}

Analogs for each SANCDB entry were extracted from the Mcule [37] and Molport [38] databases. The set of purchasable compounds in SMILES format from each of these databases (Version October 2019, latest version at that date) was downloaded. Similarity scores (Tanimoto coefficient) were computed using OpenBabel (Version 2.3) [39] fingerprint FP2 [40], a path-based fingerprint which indexes compounds linear fragments up to seven atoms. With the set of indexed fragments, a hash number from zero to 1020 was used to set a bit in a 1024bit vector. A Tanimoto coefficient of 0.6 or greater was used as cut-off for analog identification. These steps have been incorporated into an automated update pipeline in the backend which fetches updated analog data from the respective Mcule and MolPort database APIs monthly. The front end of the database has also received updates and additions to integrate information about the newly added compounds and analogs.

\section{Chemoinformatic analysis}

SANCDB compounds' scaffolds were calculated through the Bemis-Murcko decomposition of molecules [3, 25, 41-45]. Scopy [46] was utilized for scaffold calculations. Unique scaffolds from each molecule were assembled with their respective frequencies to generate the molecule cloud [46, 47].

An analysis of compound distributions into drug-like, extended drug-like, lead-like, fragment-like, proteinprotein inhibitor-like (PPI-like) subsets was performed (Table 1), using conditions defined previously [48].

To assess the coverage of SANCDB chemical space by the analogs, Principal Component Analysis (PCA) and t-Distributed Stochastic Neighbor Embedding (t-SNE) were applied on compounds' non-normalized Molecular Quantum Numbers (MQN) [49] for dimensionality reduction. MQN descriptors were computed using rdkit. Chem.rdMolDescriptors module of RDKit [50]. T-SNE is a variant of Stochastic Neighbor Embedding calculating similarity between two points in the low-dimensional space using a Student-t distribution [51]. The method has been used for chemical space analysis [52-54]. PCA and t-SNE implementations in scikit-learn were used [55, 56]. A learning rate of 100 and perplexity of 50 were used for t-SNE. Other parameters were kept to default. The 42 dimensions in MQN property space were reduced to two dimensions with both methods. The 3D structures of the identified analogs were prepared using OpenBabel [39] and resulting geometries minimized in RDKit [50] using the Merck Molecular Force Field (MMFF94) [57]. Moreover, the AutoDock ready to dock files ' $p d b q t$ ' were prepared using AutoDock 4 utilities [58]. The data was analyzed in the Jupyter Notebook [59] environment using the Python module pandas $[60,61]$ and pandas-profiling [62]. The descriptive statistics and plots were done in the same environment.

\section{Results and discussions}

\section{Database design and website interface}

SANCDB uses a MySQL database, managed by the Django framework as described previously [6]. The appearance of the site has seen numerous minor changes such as removing clutter from page elements and menus, using Bootstrap to standardize elements, and adjusting text sizes to give the site a more modern and neater appearance. Redundant and unused JavaScript and CSS libraries have been removed. This reduced the initial page load by $0.5 \mathrm{MB}$ and improved maintainability of the SANCDB codebase.

A similarity search was added to the database search functionalities. The database originally only had a substructure search function. The user can now do a similarity or a substructure search. The query is searched in the database using Open Babel FP2 fingerprints and all

Table 1 Molecular properties and conditions used to determine compound subsets

\begin{tabular}{ll}
\hline Molecular properties & Conditions \\
\hline Drug-like & MW $\leq 500 \& \mathrm{MW} \geq 150 \& \log P \leq 5 \& \mathrm{nHD} \leq 5 \& \mathrm{nHA} \leq 10$ \\
Extended drug-like & Druglike \&nRot $\leq 7 \& \mathrm{TPSA}<150$ \\
Lead-like & $\mathrm{MW} \geq 250 \& \mathrm{MW} \leq 350 \& \mathrm{nRot} \leq 7 \& \log \mathrm{P} \leq 3.5$ \\
Fragment-like & $\mathrm{nHA} \geq 3 \& \mathrm{MW} \leq 300 \& \mathrm{nHD} \leq 3 \& \log P \leq 3$ \\
PPI-like & $\mathrm{nRing} \geq 4 \& \mathrm{MW}>400 \& \mathrm{nHA}>4 \& \log P>4$
\end{tabular}

MW Molecular weight, logP lipophilicity, $n H A$ number of hydrogen bond acceptor, $n H D$ number of hydrogen bond donor, TPSA total polar surface area, $n R o t$ number of rotatable bonds and $n$ Ring number of rings 
compounds having at least 0.6 Tanimoto similarity are returned.

\section{Compound summary page and analogs}

As shown in the database schema in Fig. 1, updates featuring the molecular mass and additional compound identification parameters were implemented within the compounds table. Further, a new table was created to link analogs to the respective compounds. Each compound now has the option to view and download commercially available analogs. A total of 380,206 commercially available analogs were added from the Mcule [37] and MolPort [38] databases (at the time of writing). A user can see a list of analogs ordered by similarity score for any compound in SANCDB. Each analog links to its respective entry on the vendor's website.

From the compound summary page, for each compound there are also links to ChEMBL, DrugBank, PubChem, and ZINC databases, if available. Data points for molecular mass and, when available, a link to ChemSpider have been added to the interface. Standardized compound classifications according to ClassyFire, are

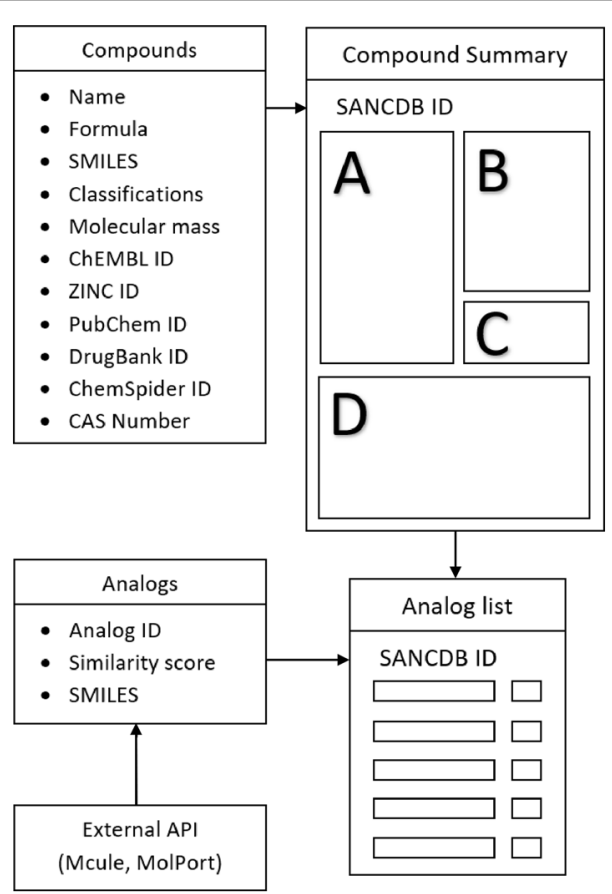

Fig. 1 Database schema detailing additions to the existing SANCDB database, compound summary and analog pages. a Basic compound information (Name, Formula, SMILES...). b Allows users to view 2D and 3D depictions of the compound. c Structure download options and navigation to page listing commercially available analogs. $\mathbf{d}$ References, classifications, other names, sources organisms and uses. Analogs are updated monthly using an automated pipeline to access external database APIs, calculate similarity scores and insert into the local SQL database displayed in addition to all existing classifications. Previously, compound structures for MOL2, PDB, minimized PDB, SDF and SMILES were available. Subsequently, Maestro and PDBQT files have been added to these options for existing and new compounds.

\section{Database content}

The database initially contained 600 compounds updated here to 1012 compounds from 359 literature references comprising mainly journal articles. Cumulatively, compounds had been isolated from 321 sources, distributed among five biological kingdoms (fungi, bacteria, plants etc.), 104 distinct families and 187 genera. The distribution of the sources of compounds according to their families and kingdoms is shown in Fig. 2a. Plants represented the majority of the sources counting for 854 compounds (78.3\%) followed by animals 219 (20.1\%), fungi $9(0.8 \%)$, chromista $6(0.5 \%)$ and finally bacteria with 3 compounds $(0.3 \%)$. This distribution is similar to some of the NPs databases where plants are the primary sources [48]. It is interesting to note the low proportion of compounds, isolated from bacterial and from microbial sources, given that they are major sources of NPs [3]. Streptomyces $s p$. with three isoflavones was the only bacteria species source reported in the entire database. Clathrina aff reticulum, Eurotium rubrum, Termitomyces microcarpus and Fusarium proliferatum were the only four fungi. Yet, microbes remain sources for major classes of antibiotics [3]. This low microbial source proportion was also consistent with other NPs databases [48,63], and some databases only focus on plants [64-66] probably because of their abundance as NP sources. The potential of NPs from microbes in South Africa may be under-explored. A similar observation was made in the source distribution of the Brazilian compound database NuBBE [48]. Plants were found to be the major producer of NPs compared to other sources (animals, fungi, bacteria) [67]. This may be explained by plant uses being more documented in traditional medicines and having easier accessibility than other sources.

Regarding families, Asparagaceae (158-14.48\%), Asteraceae $(112-10.27 \%)$, Lamiaceae $(68-6.23 \%)$, Fabaceae (67-6.14\%) and Amaryllidaceae (53-4.86\%) were the top family sources. Regarding genera, Ornithogalum (62-5.7\%), Senecio (58-5.3\%), Eucomis (39-3.6\%), Salvia (39-3.6\%) and Plocamium (38-3.5\%) were the top ones.

The top five species with the highest number of isolated compounds were Senecio pterophorus (34-3.1\%), Ornithogalum thyrsoides (27-2.4\%), Ornithogalum saundersiae (23-2.1\%), Plocamium corallorhiza (20-1.8\%) and Cephalodiscus gilchristi (19-1.7\%) as shown in Fig. 2b. Seneccio pterophorus, is an Asteraceae producing macrocyclic diester pyrrolizidine alkaloids known 


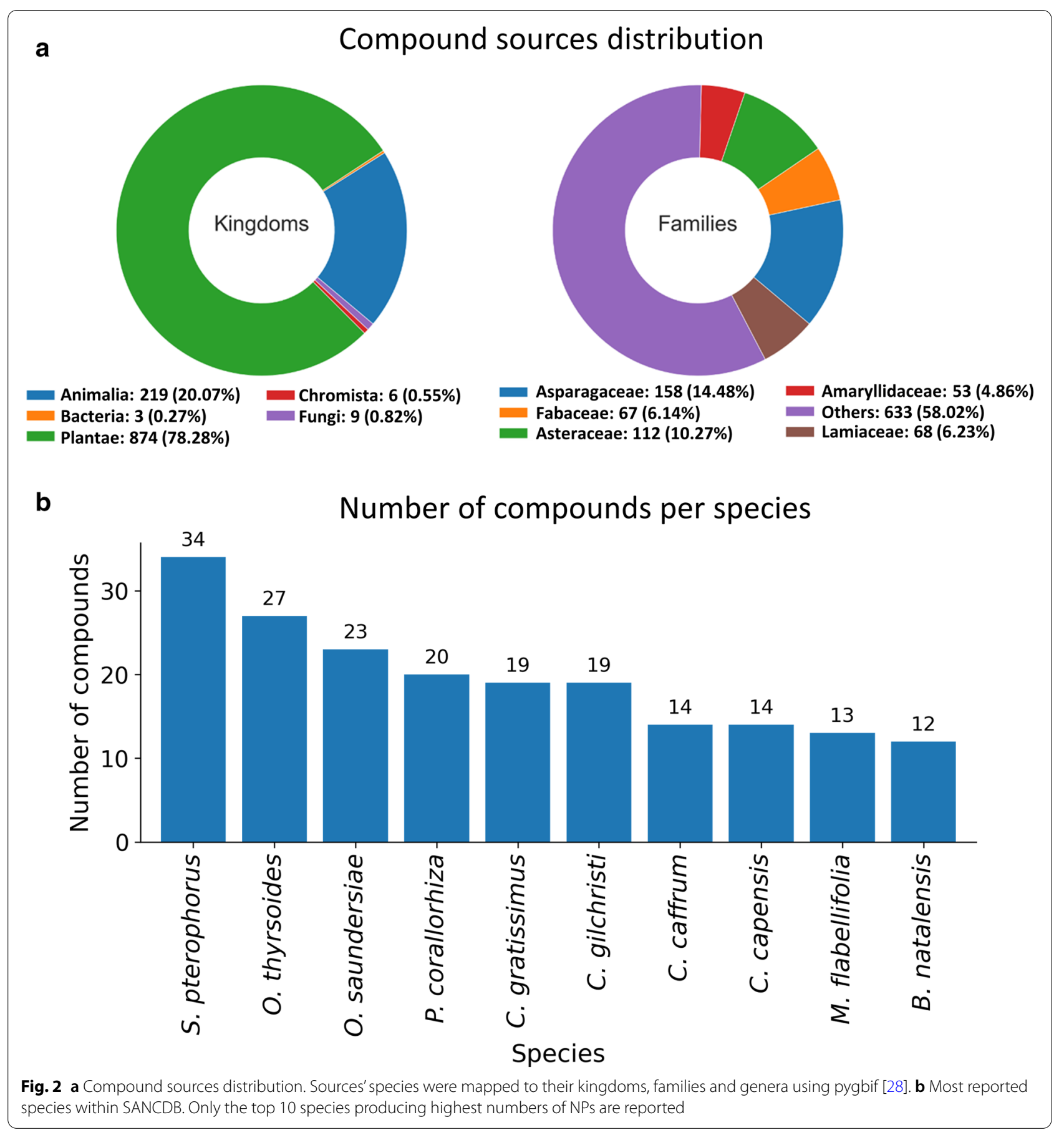

to be hepatotoxic, carcinogenic, genotoxic and teratogenic [68]. Two species of Ornithogalum plant from the Asparagaceae family: thyrsoides (27 compounds) and saundersiae (23 compounds) were the second and third sources with the highest number of isolated compounds, respectively. The thyrsoides species is widespread in South Africa, Western Cape and known for its cytotoxicity against HL-60 human promyelocytic leukemia cells
[69, 70]. Ornithogalum saundersiae is an ornamental flower from Mpumalanga, KwaZulu-Natal, and Swaziland, toxic to cattle [71, 72]. Also known as the tube worm [73], Cephalodiscus gilchristi is a cephalodiscidae containing highly potent alkaloids against lymphocytic leukemia [74]. This marine invertebrate also produces cephalostatin 1, a potent cell growth-inhibiting compound [73]. Finally, Plocamium corallorhiza is a red algae 
in the family Plocamiaceae, abundant in South Africa known for its halogenated monoterpenes [75, 76]. A common characteristic among these top sources is that they are naturally widespread. Thus, they may be more accessible for compound isolation and extraction studies and the source of more compounds as a result. Information in the database may contribute to biodiversity conservation. Indeed, the above information may contribute to finding NPs source hotspots that conservation efforts may prioritize. None of these species was found in the South African National Biodiversity Institute (SANBI) list of endangered species [77].

\section{Compounds classification}

NPs classification is useful to assess their diversity, and can be done via different schemes [48, 78]. Here, ClassyFire, which performs a hierarchical classification using structural patterns into kingdoms, superclasses, classes and subclasses, was utilized [20]. We noted 11 superclasses out of the 26 ClassyFire organic compound superclasses; 77 classes out of 764 ClassyFire classes [35]; and 124 subclasses. These numbers indicate database diversity, and therefore potentiality for a variety of biological activities. The distribution of compounds superclasses is shown in Fig. 3a, classes in Fig. 3b and molecular frameworks in Fig. 3c. Top compound classes were the phenol lipids (251-24.8\%), the steroids and steroid derivatives (141-13.9\%), the flavonoids (71-7\%), the organooxygen compounds (50-4.9\%) and the homoisoflavonoids (41-4.1\%).

Comparatively, SANCDB has a similar distribution to other compound databases. The Integrated Ethiopian Traditional Herbal Medicine and Phytochemicals Database (ETM-DB) [79] compounds classification was also done using ClassyFire, and shares the same top three superclasses. ETM-DB with 3930 compounds has 22 superclasses and 200 classes. The 500 Pan-African Natural Products Library (p-ANAPL) compounds are distributed across 30 classes [80] while the Nuclei of Bioassays, Ecophysiology and Biosynthesis of Natural Products Database (NuBBE) database has 14 classes with 2147 compounds [48]. NuBBE and p-ANAPL use however a different classification scheme. SANCDB contains $13.93 \%$ of steroids and derivatives, and was previously shown to have the highest rates of steroids compared to other NPs databases [81].

The database was rich in polycyclic compounds in Fig. 3c. Indeed, the molecular framework distribution showed that only 59 (5.8\%) of the compounds were acyclic. Nine distinct types of molecular frameworks were found in SANCDB. The most common frameworks were the aromatic heteropolycyclic (416-41.1\%), the aliphatic heteropolycyclic (232-22.9\%) and the aliphatic homopolycyclic (115-11.4\%). Molecular framework is similar to the concept of scaffold [44] and describes compounds according to their aliphaticity/aromaticity, ring count, and the diversity of atom types [35]. It is an important

\section{Compounds classification}

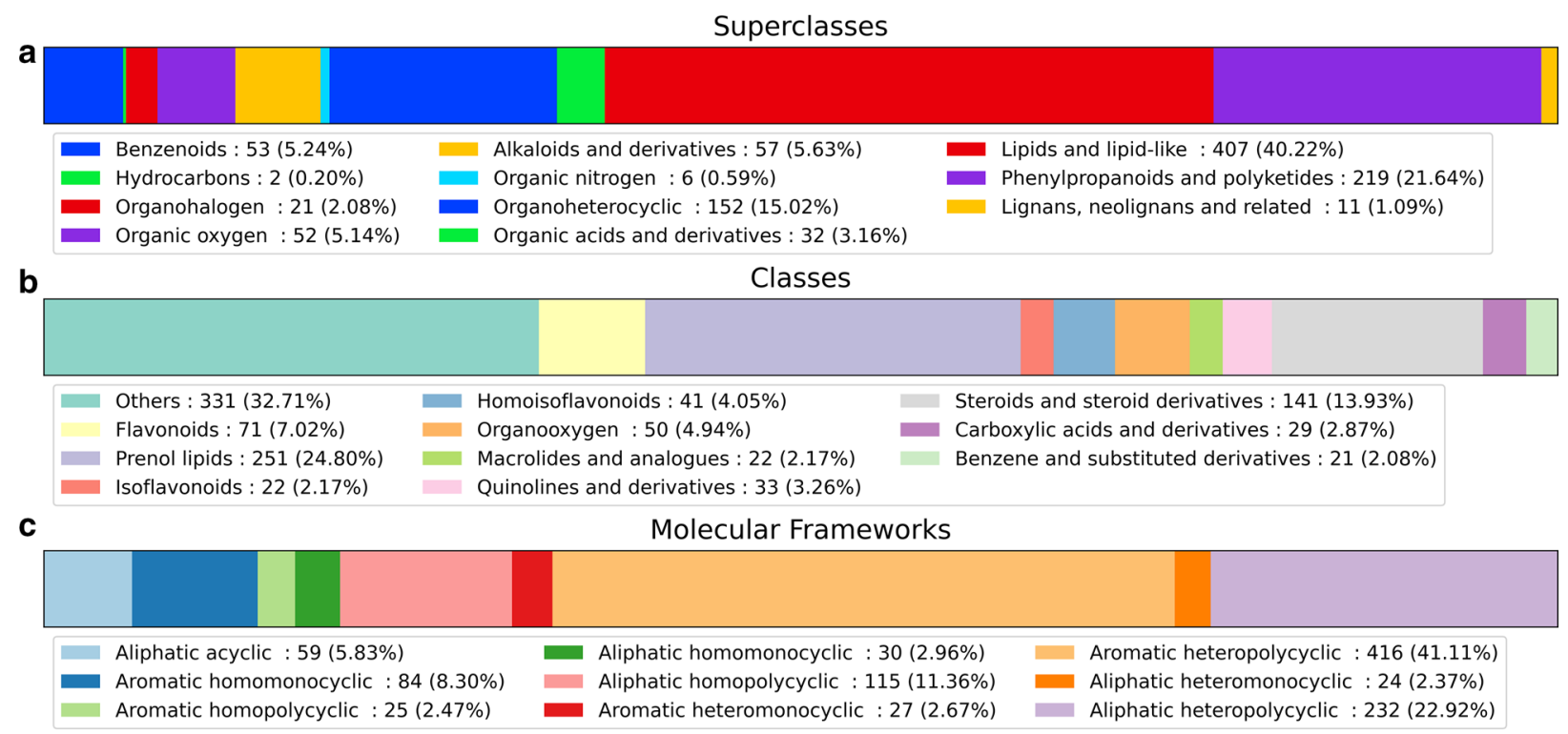

Fig. 3 Stacked bar charts of the compound classifications. a SANCDB compounds superclasses. b SANCDB compounds classes. c SANCDB molecular frameworks. Classifications were obtained from ClassyFire 
feature to assess compounds libraries' diversity in screening campaigns [26].

\section{Compounds activities}

From the source reference, where significant biological activity of isolated compounds was determined, the information was recorded and standardized to avoid duplication. The distribution of biological activities for the 318 compounds showed anticancer (158-31.6\%), antibacterial (61-12.2\%), AChE inhibitor (38-7.6\%), antimalarial, (34-6.8\%) and antiproliferative (20-4.0\%) as most common activities (Fig. 4). We noted 59 distinct activity types. Some compounds showed a variety of activities (>6 different activities): Combretastatin A-1, Ouabain, Acovenoside A, Isoorientin and Quercetin. They were also found to be associated with at least 15 predicted targets in ChEMBL [31] with 90\% confidence. They can be a good starting point for multi-target inhibitors. It is noteworthy that activities assignment was not standardized. Recorded activities could be at the molecular, cellular, tissue or disease level. Also, recording of activities was limited to only the reference in the database. Furthermore, only compounds showing a significant level of activity were recorded, limiting the number of assigned biological activities to 318 out of the 1012 in the database.

\section{Commercially available analogs}

A first evaluation of SANCDB NPs availability showed that only about $30 \%$ of SANCDB was readily purchasable. 316 were obtainable on MolPort [38] while 327 on Mcule [37]. A previous assessment of NPs commercial availability (in the ZINC subset of readily purchasable compounds) showed that only $10 \%$ were purchasable [3]. In general, NPs are insufficiently covered in commercial databases [3]. Additionally, 118 SANCDB compounds

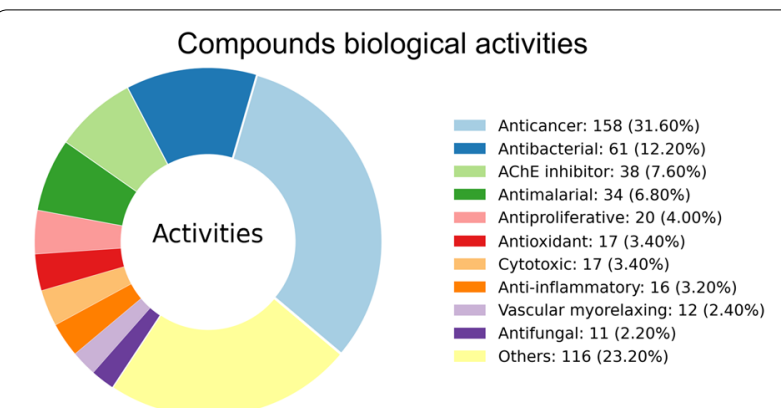

Fig. 4 SANCDB compounds biological activities. A donut chart showing the distribution of various biological activities of a total of 318 compounds which have documented use in their references in SANCDB. The top 10 activities classes are shown while the remaining ones are grouped in the others category. The biological activities of the remaining compounds in the database is yet to be reported had synthetic accessibility [82] score greater than six (see Additional file 1: Fig. S1). They may thus pose synthetic challenges [82].

In the updated version of SANCDB, a total of 380,206 unique commercially available analogs were added with an average of 1487 analogs per compound (at the time of writing). 232,747 analogs were retrieved from Mcule and 141,320 from Molport. Each compound analogs' SMILES and Tanimoto similarity scores are available for download. The downloaded Molport [38] and Mcule [37] databases contained 7,597,214 and 9,884,200 compounds, respectively in October 2019. The distribution of the number of analogs per compound is shown in Fig. 5. Frequencies ranged from 42,224 to zero analogs. SANC00428, SANC00815, SANC00656, SANC00967 and SANC00425 have the highest number of analogs with 42,224, 29,045,27,823, 26,638, 24,993 analogs respectively. These compounds have low molecular weight (MW). All compounds with more than 10,000 analogs had a MW below $300 \mathrm{Da}$. However, there was no correlation between the number of analogs and the MW with a negligible Pearson correlation of -0.09 (see Additional file 1: Figs. S2 and S3).

No analog was found for 70 compounds using a Tanimoto similarity coefficient threshold as low as 0.6 . SciFinder [27] database was used to manually retrieve analogs for these compounds. As SciFinder [27] search was per similarity interval (e.g. $0.75-0.79$ similarity interval), only the first interval having analogs, starting from the highest, was considered. The number of analogs for these compounds ranged from one to 29 with 23 compounds having only one analog [27]. The number of analogs per compounds and their respective similarity thresholds are available in Additional file 1: Table S1. Also, only 43\% (442) of the compounds had more than 1000 analogs in Molport [38] and Mcule [37] datasets. This indicates a low coverage of NPs availability considering the size of the datasets used (Molport and Mcule with $7,597,214$ and 9,884,200 respectively) and the low Tanimoto similarity coefficient cutoff used (0.6).

Analogs covered SANCDB chemical space regions (Fig. 6). SANCDB compounds formed a cluster overlapped with analogs which extend by decreasing similarity score. Analogs with similarity values in the range $(0.6,0.7)$ were the most isolated. Some SANCDB compounds without analogs occupied a small isolated cluster (zoomed region of the plot). Further analysis showed that they corresponded to compounds with zero analogs. The $\mathrm{t}$-SNE visualization showed a less dense cloud, thus indicating further separation between compounds (see Additional file 1: Fig. S4).

SANCDB analogs can expand initial drug discovery projects. Analog information is important during 
Heatmap of the number of analogs per SANCDB compound

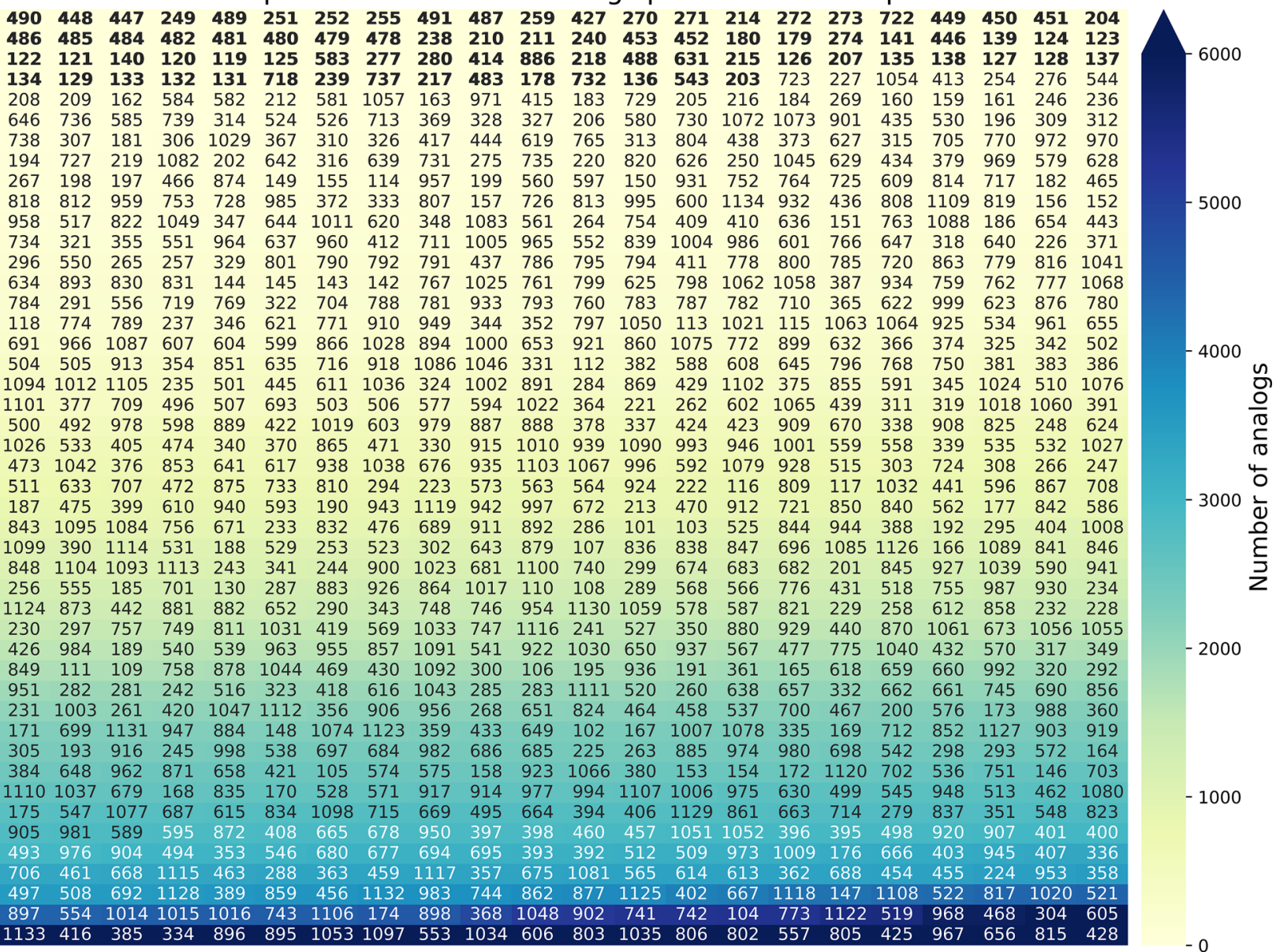

Fig. 5 Number of analogs per SANCDB compound. Compound are ordered by their number of analogs (lowest to highest, left to right). Labels on the cells are the compounds IDs. For visibility purposes, the heatmap vmax is set to 6000 . Yet some compounds have more than 6000 analogs (indicated by the arrow on the color key). IDs without analogs are in bold. Text color (compounds IDs) varies from white to black for readability. The color gradient represents the count of analogs

hit optimization in drug discovery process. Screening hits identified from SANCDB can be further optimized through their analogs $[13,16,17,19,83]$. Additionally, more potent analogs of the potential allosteric modulators identified in SANCDB [14, 15, 84] may further enhance allosteric modulation of these compounds on their targets.

\section{Scaffolds and compounds subsets}

SANCDB compounds were analyzed in terms of their scaffolds and with regard to different subsets of chemical compounds relevant to drug discovery. NPs scaffolds are of interest as they are rich in $\mathrm{sp}^{3}$-configured centers while synthetic scaffolds are generally flatter $[80,85]$. They also often serve as the basis for synthetic modifications of drug-like compounds [65]. Scaffold diversity is ideal for screening libraries as virtual screening also aims to find new scaffolds [66].

The molecule cloud visualization in Fig. 7 highlights top-ranked scaffolds. It also helps assess the diversity of scaffolds and their structural features, allowing the reader a rapid overview of the most common scaffolds [47]. However, a drawback may be the less visible of the less common scaffolds which may still be of interest. All scaffolds and their count are presented in Additional file 1: Table S2.

In SANCDB, about half of the compounds presented a unique scaffold. Indeed, 501 unique scaffolds were identified from the 1012 compounds, indicating their diversity. 59 compounds did not present a scaffold as they were purely aliphatic. Scaffold frequencies followed a "long tail" distribution (see Additional file 1: Fig. S5) common in compound datasets [47]. This shows the high number 


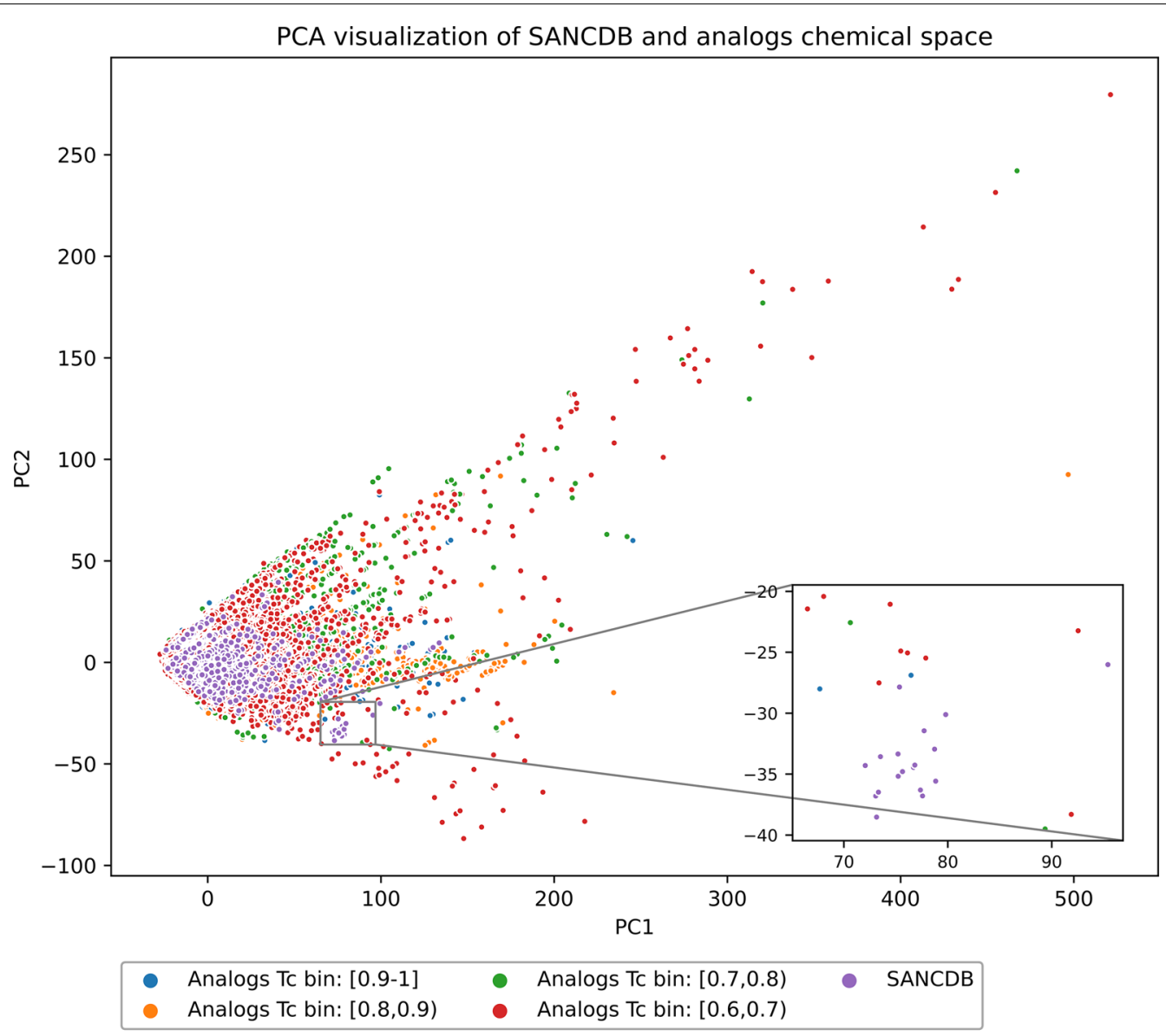

Fig. 6 PCA visualization of SANCDB and analogs chemical space. Compounds $(n=375,061)$ are represented in dots. SANCDB (violet, $n=1012)$. Analogs are in bins of similarity values: $(0.6,0.7)$ blue, $n=266,147 ;(0.7,0.8)$ orange, $n=69,336 ;(0.8,0.9)$ green, $n=24,679 ;[0.9,1]$ red, $n=13,887$. As an analog may have different similarity scores with different SANCDB compounds, the maximum similarity score was chosen for each analog. The first two components explain $81 \%$ of the variance (PC1 (66\%), PC1 (15\%))

of singletons, compounds containing a unique scaffold in the entire database, highlighting this latter diversity.

Most common scaffolds were flavonoids, already known to be common in NP datasets [44]. Interestingly, they were only the third most represented in the distribution of compound classes in Fig. 3. This may be related to a structural diversity in the first two categories compared to flavonoid which may be more homogeneous. Structures of the top 10 scaffolds are represented in Additional file 1: Fig. S6. The chromane 3-Benzylchroman-4-one was the most common scaffold with 30 compounds. Its structure presents a bicycle consisting of a 3,4-dihydro-1-benzopyran, with a ketone group which is a structural alert. Structural alerts are high reactive groups which may cause toxicity [86]. The compound is known for human monoamine oxidase B inhibition [87]. Chromane scaffolds are promiscuous in NPs [67] and known for their anticancer activity [88] which may also be related with the database richness in anticancer compounds in Fig. 4.
The second most abundant was flavone found in $21 \mathrm{com}$ pounds. Its prodrug aminoflavone reached phase 2 clinical trials for breast cancer treatment [87]. The related compound in the database may present similar activity. Finally, flavanone was the third most common scaffold with 14 compounds. This scaffold also presented a ketone group as a structural alert.

Over half of the database was drug-like or extended drug-like compounds, shown in Fig. 8. We noted a minor difference (41 compounds) between the drug-like and extended drug-like compounds, with the latter having only two more conditions to the drug-like category. PPI-like and fragments-like subsets represent the most stringent conditions for the database compounds, hence showing the least number of compounds. NP datasets may have a low proportion of fragments due to their polycyclic nature. As shown by the compound classification, SANCDB was rich in polycyclic compounds $(\sim 75 \%$ of the compounds see Fig. 3c). Thus, a low proportion of 


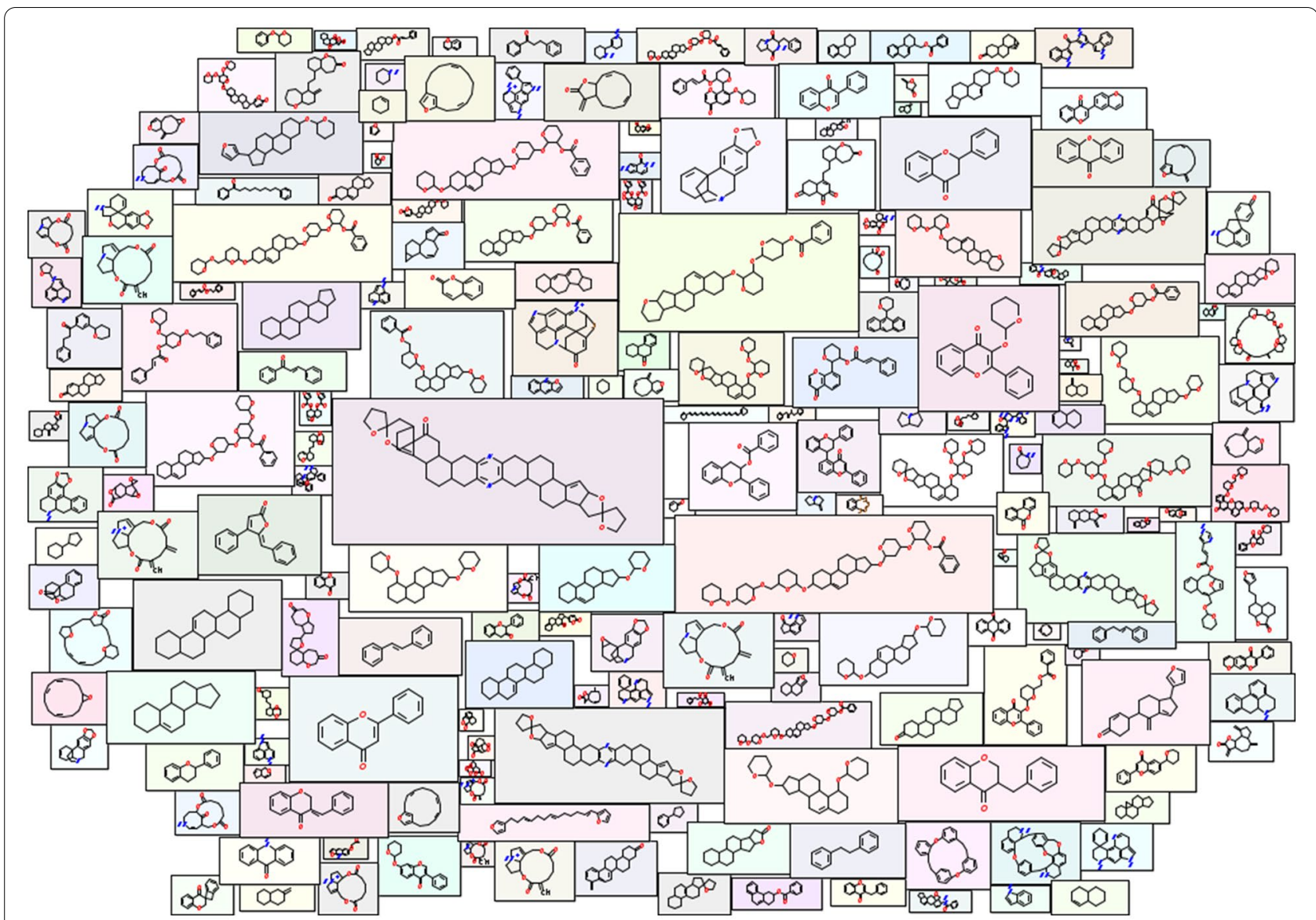

Fig. 7 Molecule cloud of SANCDB scaffolds. Structure sizes indicate scaffold frequencies. The benzene ring is a special case, being the most frequent scaffold in all large data sets [48]. Therefore, it is not displayed. A figure with the most common scaffolds and their frequencies is presented in Fig. S5

fragments is expected. PPI-like compounds $(\mathrm{MW}>400$ and $\log P>4$ ) represented the smallest set with only 78 compounds. This contrasts with the high proportion of

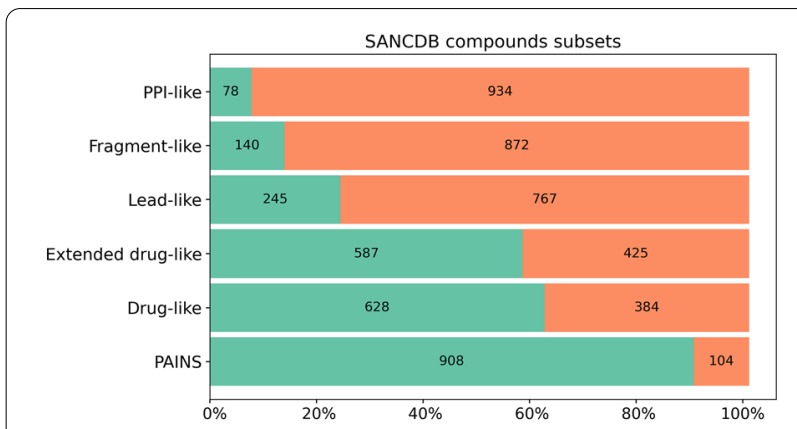

Fig. 8 Proportions of compounds in each subset (drug-like, extended drug-like, fragment-like, lead-like, PPI-like). The count of each subset and their related proportions on the $\mathrm{x}$-axis are reported. The green area indicates compounds fitting into that category. In the PAINS group, it indicates compound free of PAINS patterns polycyclic compounds in the database. Given these low proportions of fragment-like and PPI-like compounds, the database may not be suitable for fragment-based drug discovery or protein-protein inhibition.

These proportions were similar to those observed in many other NPs databases with drug-like and extended drug-like being more than $50 \%$ while PPI-like, fragmentslike and PAINS have low proportions [45].

These subsets fit different contexts in drug discovery. For example, fragments can easily be found in early stage screening to identify potent chemotypes for latter optimization. PPI-like are ideal candidates to block protein-protein interaction. The distribution of the different subsets can also be a good indicator of the ideal context for a dataset. For example, for a database enriched in fragments, fragment-based drug discovery approaches might be ideal. Small molecule databases for screening such as ZINC [33] are often subdivided into subsets. PAINS patterns are used to filter out frequent hitters in screening [89]. Hence, the various SANCDB subsets 
can be used to establish custom-made virtual screening experiments based on user's specific demands.

\section{Conclusions}

NPs remain an integral component of the drug discovery process. Hitherto, a large proportion of approved drugs have been derived from NPs [5]. This has inspired the establishment of numerous databases which contain diverse chemical classes of NPs to facilitate bioprospecting of important leads for biomedical and chemical research [1, 3]. Since the establishment of SANCDB in 2015, its usage as a source of data for both in silico screening and machine learning has been on the rise. Thus, to maintain its relevance, the current work aimed to update the database with additional compounds isolated from South African natural resources, as well as to add new functionalities aimed at providing a larger chemical space for hit exploration. To this end, the updated fully referenced relational database contains more than 1000 unique compounds from South Africa. A classification and scaffold analysis showed a diverse chemical representation with 501 unique NP scaffolds. The chemical diversity of a database is an indicator of how useful it can be for hit identification [25]. The database dataset is freely accessible and is downloadable in different chemical formats including ready to dock ones using either AutoDock [58] or Maestro from Schrödinger [37]. In consideration of the universally acknowledged limitations of NPs as a result of their complex structural organization, the current update also includes the incorporation of readily available analogs from two main commercial chemical databases (MolPort [38] and Mcule [37]). In comparison to other existing NP databases, this feature is present only in SANCDB and will provide users with a larger chemical library of compounds for both chemoinformatic and bioscreening studies. The analogs from the different databases are constantly updated via an automated pipeline making it more reliable. Analogs have been linked to their sources on Mcule [37] and MolPort [38], allowing users to obtain compounds for in vitro screening seamlessly.

\footnotetext{
Abbreviations

API: Application Programming Interface; AChE: Acetylcholinesterase; CAS: Chemical Abstracts Service; DOI: Digital Object Identifier; ETM-DB: Integrated Ethiopian Traditional Herbal Medicine and Phytochemicals Database; GBIF: Global Biodiversity Information Facility; MMFF94: Merck Molecular Force Field; MQN: Molecular Quantum Numbers; MW: Molecular weight; NaPLeS: Natural products likeness scorer; NP: Natural Product; NPASS: Natural Product Activity and Species Source; nHA: Number of hydrogen bond acceptor; nHD: Number of hydrogen bond donor; nRing: Number of rings; nRot: Number of rotatable bonds; NuBBE: Nuclei of Bioassays, Ecophysiology and Biosynthesis of Natura Products Database; p-ANAPL: Pan-African Natural Products Library; PPI-like: Protein-protein inhibitor like; RUBi: Research Unit in Bioinformatics; SANBI: South African National Biodiversity Institute; SANCDB: South African natural
}

compound database; TPSA: Total Polar Surface Area; t-SNE: T-Distributed Stochastic Neighbor Embedding.

\section{Supplementary Information}

The online version contains supplementary material available at https://doi. org/10.1186/s13321-021-00514-2.

Additional file 1: Fig S1. Distribution of SAscore for SANCDB compounds. X-axis represents SAscores and $y$-axis quantifies the corresponding probability densities. Fig. S2. Scatter plot of compounds molecular weight (MW) versus analogs count. X-axis and $y$-axis correspond to MW (Dalton) and the number of analogs respectively. Fig. S3. Scatter plot of compounds molecular weight (MW) versus analogs count. Fig. S4. t-SNE visualization of SANCDB and analogs chemical space. Fig. S5. Histogram and kernel density distribution of the scaffolds count. Fig. S6. Top 10 SANCDB scaffolds structures and their counts. Table S1. SANCDB analogs from Sci-finder for compounds without analogs on Mcule and Molport chemical databases. Table S2. A summary of all scaffold structures in SANCDB database.

\section{Acknowledgements}

Authors acknowledge use of Centre for High Performance Computing (CHPC), South Africa.

\section{Authors' contributions}

Conceptualization ÖTB; methodology, BND, MG, TMM, KL and ÖTB; validation TMM, KL and ÖTB; formal analysis, BND, MG, TMM, KL and ÖTB; investigation, BND, MG, TMM; resources, KL and ÖTB; data curation, BND, MG, TMM; writing — original draft preparation, BND, MG, TMM; writing_-review and editing, BND, MG, TMM, KL and ÖTB; visualization, BND, MG, TMM, KL and ÖTB; supervision, KL and ÖTB; project administration, KL, and ÖTB; funding acquisition ÖTB. All authors have read and agreed to the published version of the manuscript.

\section{Funding}

This work was supported through the DELTAS Africa Initiative [grant 107740/Z/15/Z]. The DELTAS Africa Initiative is an independent funding scheme of the African Academy of Sciences (AAS)'s Alliance for Accelerating Excellence in Science in Africa (AESA) and supported by the New Partnership for Africa's Development Planning and Coordinating Agency (NEPAD Agency) with funding from the Wellcome Trust [DELGEME grant 107740/Z/15/Z] and the UK government. MG is funded as a software developer by H3ABioNet, which is supported by the National Institutes of Health Common Fund grant number U24HG006941. TMM is funded as a postdoctoral fellow by Grand Challenges Africa programme [GCA/DD/rnd3/023]. Grand Challenges Africa is a programme of the African Academy of Sciences (AAS) implemented through the Alliance for Accelerating Excellence in Science in Africa (AESA) platform, an initiative of the AAS and the African Union Development Agency (AUDA-NEPAD). GC Africa is supported by the Bill \& Melinda Gates Foundation (BMGF), Swedish International Development Cooperation Agency (SIDA), German Federal Ministry of Education and Research (BMBF), Medicines for Malaria Venture (MMV), and Drug Discovery and Development Centre of University of Cape Town (H3D). The funders had no role in study design, data collection and analysis, decision to publish, or preparation of the manuscript. The content of this publication is solely the responsibility of the authors and does not necessarily represent the official views of the funders.

\section{Availability of data and materials}

All data generated or analysed during this study are included in this published article. Supplementary information accompanies this article. SANCDB is freely available at https://sancdb.rubi.ru.ac.za/.

\section{Declarations}

\section{Competing interests}

The authors declare no conflict of interest. The funders had no role in the design of the study; in the collection, analyses, or interpretation of data; in the writing of the manuscript, or in the decision to publish the results. 


\section{Author details}

${ }^{1}$ Research Unit in Bioinformatics (RUBi), Department of Biochemistry and Microbiology, Rhodes University, Makhanda/Grahamstown 6140, South Africa. ${ }^{2}$ Department of Chemistry, Rhodes University, Makhanda/Grahamstown 6140, South Africa.

Received: 21 November 2020 Accepted: 23 April 2021 Published online: 05 May 2021

\section{References}

1. Sorokina M, Steinbeck C (2020) Review on natural products databases: where to find data in 2020. J Cheminform 12:1-51. https://doi.org/10. 1186/s13321-020-00424-9

2. Mishra BB, Tiwari VK (2011) Natural products: an evolving role in future drug discovery. Eur J Med Chem 46:4769-4807. https://doi.org/10.1016/j. ejmech.2011.07.057

3. Chen Y, De Bruyn KC, Kirchmair J (2017) Data resources for the computerguided discovery of bioactive natural products. J Chem Inf Model 57:2099-2111. https://doi.org/10.1021/acs.jcim.7b00341

4. Newman DJ, Cragg GM (2020) Natural products as sources of new drugs over the nearly four decades from 01/1981 to 09/2019. J Nat Prod 83:770-803. https://doi.org/10.1021/acs.jnatprod.9b01285

5. Calixto JB (2019) The role of natural products in modern drug discovery. An Acad Bras Cienc. https://doi.org/10.1590/0001-3765201920190105

6. Hatherley R, Brown DK, Musyoka TM et al (2015) SANCDB: A South African natural compound database. J Cheminform. https://doi.org/10.1186/ s13321-015-0080-8

7. Sorokina M, Steinbeck C (2019) Naples: a natural products likeness scorer-web application and database. J Cheminform 11:1-7. https://doi. org/10.1186/s13321-019-0378-z

8. Cockroft NT, Cheng X, Fuchs JR (2019) STarFish: a stacked ensemble target fishing approach and its application to natural products. J Chem Inf Model 59:4906-4920. https://doi.org/10.1021/acs.jcim.9b00489

9. Chen Y, Stork C, Hirte S, Kirchmair J (2019) NP-Scout: machine learning approach for the quantification and visualization of the natural product likeness of small molecules. Biomolecules 9:43. https://doi.org/10.3390/ biom9020043

10. Zeng X, Zhang P, He W et al (2018) NPASS: Natural product activity and species source database for natural product research, discovery and tool development. Nucleic Acids Res. https://doi.org/10.1093/nar/gkx1026

11. Sorokina M, Merseburger $P$, Rajan K et al (2021) COCONUT online: collection of open natural products database. J Cheminform 13:2. https://doi. org/10.1186/s13321-020-00478-9

12. Veale CGL, Müller R (2020) Recent highlights in anti-infective medicinal chemistry from South Africa. ChemMedChem 15:809-826. https://doi. org/10.1002/cmdc.202000086

13. Musyoka TM, Kanzi AM, Lobb KA, Tastan Bishop Ö (2016) Structure based docking and molecular dynamic studies of plasmodial cysteine proteases against a south african natural compound and its analogs. Sci Rep 6:23690. https://doi.org/10.1038/srep23690

14. Penkler DL, Atilgan C, Tastan Bishop Ö (2018) Allosteric modulation of human hsp90a conformational dynamics. J Chem Inf Model 58:383-404. https://doi.org/10.1021/acs.jcim.7b00630

15. Amusengeri A, Tastan Bishop Ö (2019) Discorhabdin N, a South African natural compound, for Hsp72 and Hsc70 allosteric modulation: combined study of molecular modeling and dynamic residue network analysis. Molecules. https://doi.org/10.3390/molecules24010188

16. Musyoka T, Özlem TB (2019) South African abietane diterpenoids and their analogs as potential antimalarials: novel insights from hybrid computational approaches. Molecules 24:4036. https://doi.org/10.3390/ molecules24224036

17. Kimuda MP, Laming D, Hoppe HC, Tastan Bishop O (2019) Identification of novel potential inhibitors of pteridine reductase 1 in Trypanosoma brucei via computational structure-based approaches and in vitro inhibition assays. Molecules. https://doi.org/10.3390/molecules24010142

18. Karki N, Verma N, Trozzi F et al (2021) Predicting potential sars-cov-2 drugs-in depth drug database screening using deep neural network framework ssnet, classical virtual screening and docking. Int J Mol Sci 22:1-16. https://doi.org/10.3390/ijms22031392
19. Nyamai DW, Tastan Bishop Ö (2020) Identification of selective novel hits against plasmodium falciparum prolyl tRNA synthetase active site and a predicted allosteric site using in silico approaches. Int J Mol Sci 21:3803. https://doi.org/10.3390/ijms21113803

20. Fantoukh Ol, Dale OR, Parveen A et al (2019) Safety assessment of phytochemicals derived from the globalized South African Rooibos Tea (Aspalathus linearis) through interaction with CYP, PXR, and P-gp. J Agric Food Chem 67:4967-4975. https://doi.org/10.1021/acs.jafc.9b00846

21. Awolola GV, Sofidiya MO, Baijnath $\mathrm{H}$ et al (2019) The phytochemistry and gastroprotective activities of the leaves of Ficus glumosa. South African J Bot 126:190-195. https://doi.org/10.1016/j.sajb.2019.01.015

22. Engelhardt C, Petereit F, Lechtenberg M et al (2016) Qualitative and quantitative phytochemical characterization of Myrothamnus flabellifolia Welw. Fitoterapia 114:69-80. https://doi.org/10.1016/j.fitote.2016.08.013

23. Bernardini S, Tiezzi A, Laghezza Masci V, Ovidi E (2018) Natural products for human health: an historical overview of the drug discovery approaches. Nat Prod Res 32:1926-1950. https://doi.org/10.1080/14786 419.2017.1356838

24. Cragg GM, Newman DJ (2013) Natural products: a continuing source of novel drug leads. Biochim Biophys Acta 1830:3670-3695. https://doi.org/ 10.1016/j.bbagen.2013.02.008

25. Garcia-Castro M, Zimmermann S, Sankar MG, Kumar K (2016) Scaffold diversity synthesis and its application in probe and drug discovery. Angew Chemie Int Ed 55:7586-7605. https://doi.org/10.1002/anie.20150 8818

26. Elsevier (2010) Elsevier Developer Portal. In: Elsevier.com. https://dev.elsev ier.com/tecdoc text mining.html. Accessed 11 Jul 2020

27. CAS (2015) SciFinder - A CAS Solution. In: Publication. http://www.cas. org/products/scifinder. Accessed 24 Oct 2015

28. Chamberlain S pygbif 0.4.0 documentation-pygbif 0.4.0 documentation. https://pygbif.readthedocs.io/en/latest/index.html. Accessed 4 Jun 2020

29. GBIF. https://www.gbif.org/. Accessed 4 Jun 2020

30. Swain M (2014) PubChemPy: A way to interact with PubChem in Python

31. Mendez D, Gaulton A, Bento AP et al (2019) ChEMBL: towards direct deposition of bioassay data. Nucleic Acids Res 47:D930-D940. https://doi. org/10.1093/nar/gky1075

32. Lo EJ, lynkkaran I, Li C et al (2017) DrugBank 5.0: a major update to the DrugBank database for 2018. Nucleic Acids Res 46:D1074-D1082. https:// doi.org/10.1093/nar/gkx1037

33. Sterling T, Irwin JJ (2015) ZINC 15-ligand discovery for everyone. J Chem Inf Model 55:2324-2337. https://doi.org/10.1021/acs.jcim.5b00559

34. Kim S, Chen J, Cheng T et al (2019) PubChem 2019 update: improved access to chemical data. Nucleic Acids Res 47:D1102-D1109. https://doi. org/10.1093/nar/gky1033

35. Djoumbou Feunang Y, Eisner R, Knox C et al (2016) ClassyFire: automated chemical classification with a comprehensive, computable taxonomy. J Cheminform 8:1-20. https://doi.org/10.1186/s13321-016-0174-y

36. Release S (2017) 1: Maestro. Schrödinger LLC, New York, p 2017

37. Kiss R, Sandor M, Szalai FA (2012) http://Mcule.com: a public web service for drug discovery. J Cheminform. DOl:https://doi.org/10.1186/ 1758-2946-4-s1-p17

38. Easy compound ordering service - MolPort. https://www.molport.com/ shop/index. Accessed 11 Jul 2020

39. O'Boyle NM, Banck M, James CA et al (2011) Open babel: an open chemical toolbox. J Cheminform 3:33. https://doi.org/10.1186/1758-2946-3-33

40. Molecular fingerprints and similarity searching-Open Babel v2.3.0 documentation. http://openbabel.org/docs/dev/Features/Fingerprints. html. Accessed 20 Sep 2020

41. Pilón-Jiménez BA, Saldívar-González FI, Díaz-Eufracio BI, Medina-Franco JL (2019) BIOFACQUIM: a Mexican compound database of natural products. Biomolecules. https://doi.org/10.3390/biom9010031

42. Kearney SE, Zahoránszky-Kőhalmi G, Brimacombe KR et al (2018) Canvass: a crowd-sourced, natural product screening library for exploring biological space. ACS Cent Sci. https://doi.org/10.26434/CHEMRXIV. 7172369.V2

43. Sánchez-Cruz N, Pilón-Jiménez BA, Medina-Franco JL (2020) Functional group and diversity analysis of BIOFACQUIM: a Mexican natural product database. F1000Res 8:2071. https://doi.org/10.12688/f1000resea rch.21540.2 
44. Singh N, Guha R, Giulianotti MA et al (2009) Chemoinformatic analysis of combinatorial libraries, drugs, natural products, and molecular libraries small molecule repository. J Chem Inf Model 49:1010-1024 https://doi.org/10.1021/ci800426u

45. Saldívar-Gonzaíez FI, Valli M, Andricopulo AD et al (2019) Chemical space and diversity of the NuBBE database: a chemoinformatic characterization. J Chem Inf Model. https://doi.org/10.1021/acs.jcim.8b00619

46. The Scopy's documentation-Scopy 1.2.3 documentation. https:// scopy.iamkotori.com/index.html. Accessed 10 Aug 2020

47. Ertl P, Rohde B (2012) The molecule cloud-compact visualization of large collections of molecules. J Cheminform 4:1. https://doi.org/10. 1186/1758-2946-4-12

48. Pilon AC, Valli M, Dametto AC et al (2017) NuBBEDB: An updated database to uncover chemical and biological information from Brazilian biodiversity. Sci Rep. https://doi.org/10.1038/s41598-017-07451-x

49. Nguyen KT, Blum LC, Van Deursen R, Reymond JL (2009) Classification of organic molecules by molecular quantum numbers. ChemMedChem 4:1803-1805. https://doi.org/10.1002/cmdc.200900317

50. Landrum G (2016) RDKit: open-source cheminformatics software

51. Van Der Maaten L, Hinton G (2008) Visualizing data using t-SNE. J Mach Learn Res 9:2579-2625

52. Janssen AP, Grimm SH, Wijdeven MRH et al (2018) Drug discovery maps, a machine learning model that visualizes and predicts kinomeinhibitor interaction landscapes. J Chem Inf Model. https://doi.org/10. 1021/acs.jcim.8b00640

53. Naveja JJ, Medina-Franco JL (2019) Finding constellations in chemical space through core analysis. Front Chem 7:510. https://doi.org/10. 3389/fchem.2019.00510

54. Yosipof A, Guedes RC, García-Sosa AT (2018) Data mining and machine learning models for predicting drug likeness and their disease or organ category. Front Chem 6:162. https://doi.org/10.3389/fchem.2018.00162

55. Pedregosa F, Varoquaux GG, Gramfort A et al (2011) Scikit-learn: Machine learning in Python. J Mach Learn Res 12:2825-2830

56. sklearn.manifold.TSNE—scikit-learn 0.23.1 documentation. https:// scikit-learn.org/stable/modules/generated/sklearn.manifold.TSNE.html. Accessed 17 Jul 2020

57. Tosco P, Stiefl N, Landrum G (2014) Bringing the MMFF force field to the RDKit: Implementation and validation. J Cheminform 6:37. https:// doi.org/10.1186/s13321-014-0037-3

58. Morris GM, Huey R, Lindstrom W et al (2009) AutoDock4 and AutoDockTools4: automated docking with selective receptor flexibility. J Comput Chem 30:2785-2791. https://doi.org/10.1002/jcc.21256

59. Kluyver T, Ragan-Kelley B, Pérez F, et al (2016) Jupyter Notebooks-a publishing format for reproducible computational workflows. In: ELPUB. pp 87-90

60. McKinney W (2011) pandas: a Foundational python library for data analysis and statistics. Python High Perform Sci Comput 1-9.

61. McKinney W (2010) Data structures for statistical computing in Python. Proc 9th Python Sci Conf. https://doi.org/10.25080/majora-92bf1 922-00a

62. Brugman S (2019) pandas-profiling: exploratory data analysis for Python

63. Ntie-Kang F, Telukunta KK, Döring K et al (2017) NANPDB: a resource for natural products from northern African sources. J Nat Prod 80:20672076. https://doi.org/10.1021/acs.jnatprod.7b00283

64. Ntie-Kang F, Zofou D, Babiaka SB et al (2013) AfroDb: a select highly potent and diverse natural product library from African medicinal plants. PLoS ONE. https://doi.org/10.1371/journal.pone.0078085

65. Ntie-Kang F, Onguéné PA, Scharfe M et al (2014) ConMedNP: a natura product library from Central African medicinal plants for drug discovery. RSC Adv 4:409-419. https://doi.org/10.1039/c3ra43754j

66. Ntie-Kang F, Mbah JA, Mbaze LMa et al (2013) CamMedNP: Building the Cameroonian 3D structural natural products database for virtual screening. BMC Complement Altern Med. https://doi.org/10.1186/ 1472-6882-13-88

67. Ertl P, Schuhmann T (2020) Cheminformatics analysis of natural product scaffolds: comparison of scaffolds produced by animals, plants, fungi and bacteria. Mol Inform. https://doi.org/10.1002/minf.20200 0017

68. Castells E, Mulder PPJ, Pérez-Trujillo M (2014) Diversity of pyrrolizidine alkaloids in native and invasive Senecio pterophorus (Asteraceae):
Implications for toxicity. Phytochemistry 108:137-146. https://doi.org/ 10.1016/j.phytochem.2014.09.006

69. Kuroda M, Ori K, Mimaki Y (2006) Ornithosaponins A-D, four new polyoxygenated steroidal glycosides from the bulbs of Ornithogalum thyrsoides. Steroids 71:199-205. https://doi.org/10.1016/j.steroids. 2005.10.001

70. Ornithogalum thyrsoides | PlantZAfrica. http://pza.sanbi.org/ornithogal um-thyrsoides. Accessed 15 Jun 2020

71. Ornithogalum saundersiae / PlantZAfrica. http://pza.sanbi.org/ornit hogalum-saundersiae. Accessed 15 Jun 2020

72. Iguchi T, Kuroda M, Naito R et al (2019) Cholestane glycosides from Ornithogalum saundersiae bulbs and the induction of apoptosis in $\mathrm{HL}-60$ cells by OSW-1 through a mitochondrial-independent signaling pathway. J Nat Med 73:131-145. https://doi.org/10.1007/ s11418-018-1252-4

73. Davies-Coleman M, Veale C (2015) Recent advances in drug discovery from South African marine invertebrates. Mar Drugs 13:6366-6383. https://doi.org/10.3390/md13106366

74. Pettit GR, Kamano Y, Dufresne C et al (1989) Isolation and structure of the unusual Indian Ocean Cephalodiscus gilchristi components, cephalostatins 5 and 6. Can J Chem 67:1509-1513. https://doi.org/10. 1139/v89-231

75. Knott MG, Mkwananzi H, Arendse CE et al (2005) Plocoralides A-C, polyhalogenated monoterpenes from the marine alga Plocamium corallorhiza. Phytochemistry 66:1108-1112. https://doi.org/10.1016/j. phytochem.2005.03.029

76. Mann MGA, Mkwananzi HB, Antunes EM et al (2007) Halogenated monoterpene aldehydes from the South African marine alga Plocamium corallorhiza. J Nat Prod 70:596-599. https://doi.org/10.1021/ np060547c

77. SANBI (2019) Threatened Species Programme / SANBI Red List of South African Plants. In: South African Natl Biodivers Inst http://redlist.sanbi. org/stats.php. Accessed 9 Jul 2020

78. Banerjee P, Erehman J, Gohlke BO et al (2015) Super natural II-a database of natural products. Nucleic Acids Res. https://doi.org/10.1093/ nar/gku886

79. Bultum LE, Woyessa AM, Lee D (2019) ETM-DB: integrated Ethiopian traditional herbal medicine and phytochemicals database. BMC Complement Altern Med. https://doi.org/10.1186/s12906-019-2634-1

80. Ntie-Kang F, Amoa Onguéné P, Fotso GW et al (2014) Virtualizing the p-ANAPL Library: a step towards drug discovery from African medicinal plants. PLoS ONE 9:e90655. https://doi.org/10.1371/journal.pone.00906 55

81. Chen Y, Garcia De Lomana M, Friedrich NO, Kirchmair J (2018) Characterization of the chemical space of known and readily obtainable natural products. J Chem Inf Model 58:1518-1532. https://doi.org/10. 1021/acs.jcim.8b00302

82. Ertl P, Schuffenhauer A (2009) Estimation of synthetic accessibility score of drug-like molecules based on molecular complexity and fragment contributions. J Cheminform 1:8. https://doi.org/10.1186/ 1758-2946-1-8

83. Elkhattabi L, Charoute H, Saile R, Barakat. (2020) A computational approach revealed potential affinity of antiasthmatics against receptor binding domain of 2019n-cov spike glycoprotein. https://doi.org/10. 26434/chemrxiv.12115638.v1

84. Nyamai DW, Tastan Bishop Ö (2019) Aminoacyl tRNA synthetases as malarial drug targets: a comparative bioinformatics study. Malar J 18:1-27. https://doi.org/10.1186/s12936-019-2665-6

85. Meyers J, Carter M, Mok NY, Brown N (2016) On the origins of threedimensionality in drug-like molecules. Future Med Chem 8:1753-1767. https://doi.org/10.4155/fmc-2016-0095

86. Limban C, Nută DC, Chiriță C et al (2018) The use of structural alerts to avoid the toxicity of pharmaceuticals. Toxicol Reports 5:943-953

87. CHEMBL1766622 Compound Report Card. https://www.ebi.ac.uk/ chembldb/index.php/compound/inspect/CHEMBL2079699. Accessed 3 Sep 2020

88. Simon L, Abdul Salam AA, Madan Kumar S et al (2017) Synthesis, anticancer, structural, and computational docking studies of 3-benzylchroman-4-one derivatives. Bioorganic Med Chem Lett 27:5284-5290. https://doi.org/10.1016/j.bmcl.2017.10.026 
89. Baell JB, Holloway GA (2010) New substructure filters for removal of pan assay interference compounds (PAINS) from screening libraries and for their exclusion in bioassays. J Med Chem 53:2719-2740. https://doi.org/ 10.1021/jm901137j

\section{Publisher's Note}

Springer Nature remains neutral with regard to jurisdictional claims in published maps and institutional affiliations.
Ready to submit your research? Choose BMC and benefit from:

- fast, convenient online submission

- thorough peer review by experienced researchers in your field

- rapid publication on acceptance

- support for research data, including large and complex data types

- gold Open Access which fosters wider collaboration and increased citations

- maximum visibility for your research: over $100 \mathrm{M}$ website views per year

At BMC, research is always in progress.

Learn more biomedcentral.com/submissions 